\title{
Hemangioma Colorretal
}

\author{
Colon Rectal Hemangioma
}

\author{
JOÃO BATISTAPINHEIRO BARRETO ${ }^{1}$; JOAQUIM DAVID CARNEIRO NETO NIKOLAY COELHO DAMOTA $^{3}$; \\ SYOMARAPEREIRADACOSTAMELO ${ }^{4}$.
}

\author{
Chefe do Serviço de Coloproctologia do Hospital Universitário Presidente Dutra - HUUFMA. \\ Hospital Universitário Presidente Dutra ${ }^{l}$; Médico. Universidade Federal do Maranhão. HUUFMA²; Médico. Residente \\ de Cirurgia. HUUFMA'; Médica. Patologista. HUUFMA4.
}

BARRETO JBP; CARNEIRO NETO JD; MOTA NC; MELO SPC. Hemangioma Colorretal. Rev bras Coloproct, 2007;27(2): 210-213.

RESUMO: O hemangioma colorretal (HCR) é uma lesão vascular benigna rara, com manifestação clínica geralmente entre 5 e 25 anos de idade. Faz parte do diagnóstico diferencial das causas de hemorragia digestiva baixa, sendo confundido, na maioria das vezes, com entidades mais comuns, como hemorróidas e doenças inflamatórias intestinais. O retardo do diagnóstico ocorre freqüentemente devido ao desconhecimento da doença, com taxas de mortalidade alcançando 40 a $50 \%$ na presença de sangramento importante. $O$ caso relatado é de uma paciente de 17 anos de idade, admitida no Serviço de Colo-proctologia do Hospital Universitário - HUUFMA, em setembro de 2005, com anemia e sangramento retal, desde a infância, de forma intermitente e não dolorosa. Apresentado sua história clínica e propedêutica diagnóstica, realizada por meio de exames laboratoriais, endoscopia digestiva alta, colonoscopia e arteriografia de mesentéricas e ilíacas internas. $O$ tratamento cirúrgico realizado foi retossigmoidectomia convencional com anastomose colorretal baixa, com boa evolução pós-operatória, tendo o exame histopatológico da peça cirúrgica ressecada, confirmado o diagnostico.

Descritores: Hemangioma colorretal, hemorragia digestiva baixa.

\section{INTRODUÇÃO}

Considera-se como Hemorragia Digestiva Baixa (HDB) o sangramento que ocorre devido à lesão estar situada distalmente ao ângulo de Treitz. Das causas de hemorragia do trato gastrointestinal, a HDB é responsável por $15 \%$, sendo o intestino grosso a origem em 95 a 97\%. Em pacientes com idade inferior a 50 anos a principal etiologia é de origem anorretal, enquanto naqueles com idade acima de 70 anos é a doença diverticular ${ }^{1,2}$.

Hemangioma colorretal é uma causa rara de HDB. Sua principal manifestação clínica é sangramento retal indolor, que ocorre geralmente desde a infância, com diagnóstico feito frequientemente na idade adulta.

A colonoscopia leva a suspeita diagnóstica, sem realização de biópsia. Arteriografias de artérias de mesentéricas e ilíacas internas são feitas de forma complementar.

O tratamento indicado é a ressecção do segmento afetado, sempre que possível com preservação dos esfíncteres anais.

\section{RELATO DO CASO}

Paciente com 17 anos de idade, sexo feminino, natural do interior do estado, sendo admitida no serviço de Colo-proctologia do Hospital Universitário Presidente Dutra (HUPD), com história de anemia e hematoquezia desde a infância, de forma intermitente e não dolorosa, eventualmente com tonteira e astenia. Ao exame físico, apresentava-se descorada $(++/ 4+)$, eupnéica, sem lesões muco-cutâneas. Abdome indolor à palpação e sem visceromegalias. Exame proctológico:

Trabalho realizado na Universidade Federal do Maranhão - UFMA, Hospital Universitário Presidente Dutra. Serviço de Coloproctologia. São Luís, Maranhão, Brasil.

Recebido em 26/10/2006

Aceito para publicação em 12/12/2006 
inspeção e toque retal normais. A investigação diagnóstica inicial foi feita com a realização de colonoscopia, evidenciando-se ectasias vasculares no colón sigmóide e reto, atingindo em alguns locais quase toda a circunferência da parede do intestino grosso, suspeitando-se de hemangioma colorretal (Figura 1).

A endoscopia digestiva alta, arteriografia de mesentéricas superior e inferior e ilíacas internas, foram normais (Figura 2).

Com a suspeita de hemangioma colorretal, foi indicado tratamento cirúrgico. Paciente apresentavase anêmica ( Hb: 6g/dl, Ht: 20\%); coagulograma, funções hepática e renal normais. Foram transfundidas quatro unidades de concentrado hemácia no pré-ope-

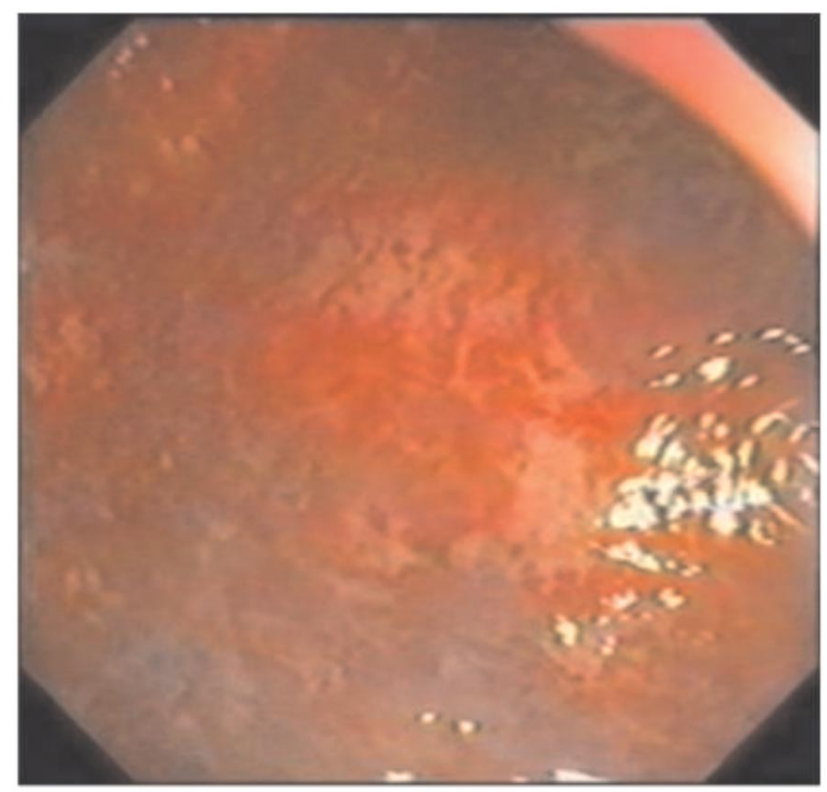

Figura 1 - Colonoscopia: evidencias de ectasias vasculares em região do colón, sigmóide e reto. ratório. Foi realizado retossigmoidectomia com anastomose colorretal baixa, com uso do grampeador circular de $29 \mathrm{~mm}$. Os achados cirúrgicos são mostrados na figura 3 .

A evolução pós-operatória foi satisfatória, tendo a paciente recebido alta no $7^{\circ}$ dia, com dieta normal e sem queixa de sangramento retal.

O exame anátomo-histopatólogico demonstrou aumento de espessura de todas as camadas da parede do reto e sigmóide; submucosa com vasos dilatados e congestos, alguns com trombos e flebólitos, confirmando o diagnóstico de hemangioma colorretal (Figuras 4 e 5).

\section{DISCUSSÃO}

Embora a apresentação clínica do hemangioma colorretal seja típica, com sangramento retal indolor

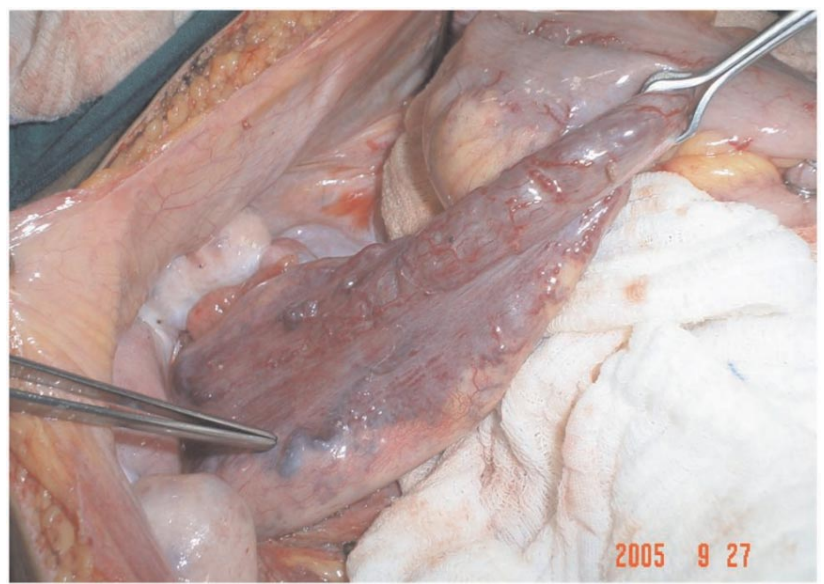

Figura 3 - Achado cirúrgico: Ectasias vasculares em Sigmóide.

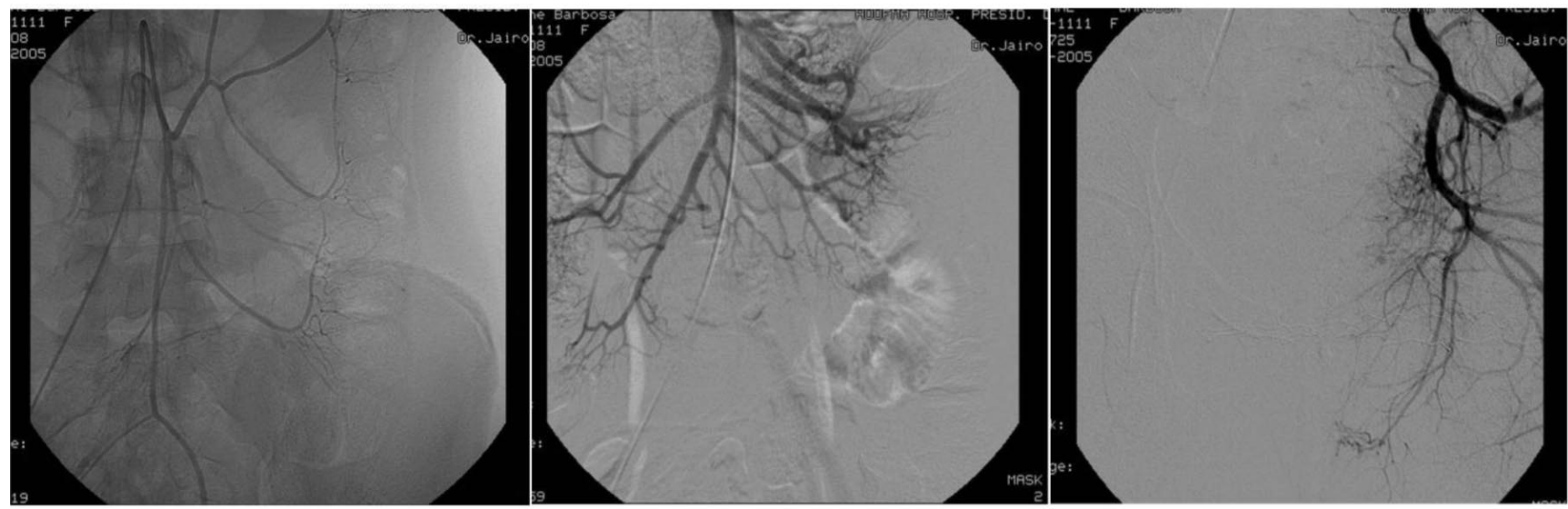

Figura 2 - Arteriografia de arterias mesentéricas e ilíacas internas: Sem alterações. 


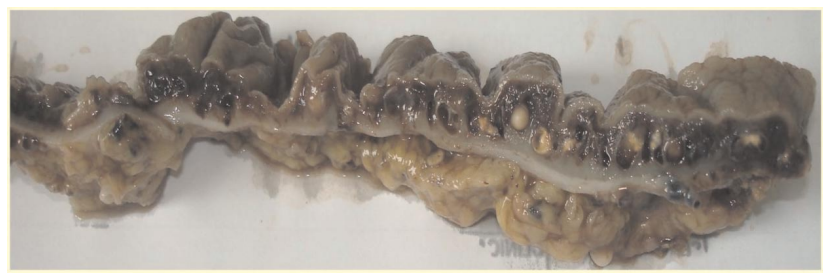

Figura 4 - Peça cirúrgica enviada para exame anátomohistopatológico: presença de flebolitos.

desde a infância, o diagnóstico geralmente não é feito antes de 30 anos de idade. Em geral, pacientes portadores de hemangiomas são submetidos a intervenções cirúrgicas desnecessárias por doenças diagnosticadas incorretamente, como hemorróidas e doenças inflamatórias ${ }^{3}$

Apesar do hemangioma colorretal raramente estar associado a determinadas síndromes (KlippelTrenaunay, Osler-Weber-Rendu e Maffuci), hemangiomas cutâneos, encondromas, epistaxe, hipertrofia do tecido ósseo e de partes moles devem ser pesquisados. Na presença de uma destas síndromes,pode ser tomada conduta diferente da aplicada ao hemangioma isolado ${ }^{4,5}$.

O diagnóstico do hemangioma colorretal é suspeitado na colonoscopia, com presença de ectasias vasculares. Biópsia geralmente não é realizada pelo risco de sangramento ${ }^{6}$.

Arteriografia de mesentéricas e ilíacas internas podem identificar a existência de sangramentos

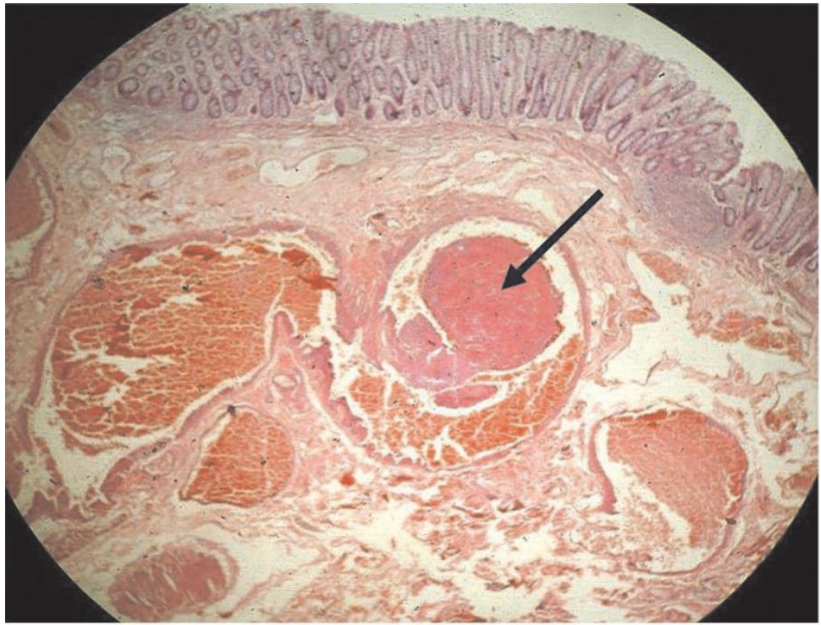

Figura 5 - Exame histopatológico: Formação de trombo em vaso da submucosa (seta).

ativos e delimitar a extensão da massa tumoral vascular. Porém, na maioria dos casos, o exame não é conclusivo ou normal ${ }^{7}$.

O hemangioma colorretal é classificado em dois tipos: polipóide e não-polipóide. O segundo, que é a forma difusa, encontrado na ampla maioria dos casos, tem como tratamento indicado a ressecção do segmento afetado. Quando da localização no sigmóide e no reto realiza-se a anastomose colo-retal baixa ou colo-anal, buscando-se a preservação dos esfíncteres anais. Excisões endoscópicas podem ser utilizadas no tratamento de lesões polipóides pequenas, constituindo tratamento de exceção ${ }^{8}$.

\begin{abstract}
The colon and rectum hemangioma is a rare benign vascular lesion, with clinical features usually between 5 and 25 years of age. It is included in the differential diagnose of the lower digestive bleeding causes, and has been frequently misdiagnosed with other more common entities, like hemorrhoids and bowel inflammatory disease. The late diagnose occurs usually because of the rarity of the disease, with mortality rates reaching 40 to $50 \%$ in presence of severe bleeding. We report a case of a 17 years old girl who was admitted at the Coloproctology Service of the Academic Hospital - HUUFMA, in September 2005, with anemia and intermittent rectal bleeding since childhood. Laboratorial findings included laboratorial exams, GI endoscopy, colonoscopy and arteriography of mesenteric and internal iliacs arteries. Conventional rectosigmoidectomy with low colorectal anastomosis was done, with good postoperative evolution and histopathologic examination of the resected specimen.
\end{abstract}

Keywords: Colorectal hemangioma, lower digestive bleeding.

\section{REFERÊNCIAS}

1. Fontes, PRO; Matos, AA; Zamin Jr., I. Hemorragia Digestiva Baixa. In: Coelho, JCU. Aparelho Digestivo: clínica e cirurgia.2004 3rd Ed. São Paulo. p 145-160.

2. Bass, BL; Turner, DJ. Acute Gastrintestinal. In: Sabiston. Textbook of surgery. 17th Ed. Saunders; 2004. p 1241-1264.
3. Marie L. Borum, MD, MPH. Cavernous Colorectal Hemangioma: A Rare Case of Gastrintestinal Bleeding and a Review of Literature. Digestive Diseases and Sciences. 1997; 42 (12): p 2468-2470. December, 1997

4. Kotze P G, Soares A V, Lima MC, Baldin- Junior A, Sartor MA. Síndrome Klippel - Trenaunay: Uma Causa Rara de Hemorragia Digestiva Baixa. Rev bras Coloproct. 2002; 22 (2): p 109-112, 2002. 
5. Perry WH. Clinical spectrum of hereditary hemorrhagic telangiectasia (Osler-Weber-Rendu). Am J Med 1987; 82: p 985-997

6. Kyoko Yorozuya, Masahiko Watanabe, Hirotoshi Hasegawa, et al. Diffuse Cavernous Hemangioma of the Rectum: Report of a Case. Surgery Today. 2003; 33: p 309-311.

7. Almeida, EC et al. Hemangioma de reto. Rev bras Coloproct. 1988; 8 (2): p 72-75. Abril-Junho, 1988

8. Li- ching Liang, M D. Forbas N, David J, et al. Endoscopy polipectomy of an unsually long polypoid colorectal cavernous hemangioma. Gastrintestinal endoscopy. 1998; 47 (3).

9. Tan TCF, Wang JY, Cheng YC, Wan WYL.Diffuse Cavernous Hemangioma of the Rectum Complicated by Invasion of Pelvic
Estructures: Report of Two Cases. Dis Colon Rectum. 1998; 41: p 1062-1066. August 1998.

10. H.T Wang.Y.Tu. Diffuse cavernous hemangioma of the rectosigmoid Colon. Tech Colo proctol. 2005; 9: p 145-148.

\section{Endereço para correspondência:}

JOÃO BATISTA PINHEIRO BARRETO

CPM - Clínica de Proctologia do Maranhão

Renascença Medical Center

Av. Collares Moreira, 11 QD. 23

$5^{\circ}$ Andar - Renascença II

Fone/Fax: (98) 3227-4332

E-mail:jbpbarreto@uol.com.br; jdavidneto@hotmail.com 